\title{
Ectopic Expression of Fiber Related Gbwril Complements Seed Phenotype in Arabidopsis thaliana
}

\author{
Mahnoor Imran $^{1}$, Shaher Bano ${ }^{1}$, Asma Manzoor ${ }^{2}$, Muhammad Azeem $^{3 *}$, Tanzeela Rehman ${ }^{1}$, Samina Yousaf $^{4}$, Maria \\ But $^{1}$, Tousif Hussain ${ }^{5}$ and Uzma Qaisar ${ }^{1 *}$ \\ ${ }^{1}$ School of Biological Sciences, University of the Punjab, Lahore, Pakistan \\ ${ }^{2}$ Institute of Biochemistry and Biotechnology, University of the Punjab, Lahore, Pakistan \\ ${ }^{3}$ Botany Department, Government College University, Faisalabad, Pakistan \\ ${ }^{4}$ Botany Department, University of the Punjab, Lahore, Pakistan \\ ${ }^{5}$ Centre for Advanced Studies in Physics, Government College University, Lahore, Pakistan \\ *For correspondence: uzma.sbs@pu.edu.pk; mazeem@gcuf.edu.pk \\ Received 07 July 2020; Accepted 17 September 2020; Published 10 December 2020
}

\begin{abstract}
WRINKLED1 belongs to AP2/EREB family of transcription factors whose role has been well established in seed oil biosynthesis. The objective of the study was to trace the role of fiber related Gbwril in seed development and fatty acid biosynthesis. In this study, we isolated a transcript from elite fiber producing cotton (Gossypium barbadense), which is overexpressed in $G$. barbadense fibers as compared to $G$. hirsutum and G. arboreum. The putative protein encoded by this transcript exhibited homology in specific domains and protein structure with WRINKLED1 of Arabidopsis thaliana and was thus designated as Gbwril. In this study, we investigated the functional homology of fiber elongation related Gbwril with fatty acid biosynthesis regulator Atwril. Ectopic expression of Gbwril in wri1-3 mutant of A. thaliana was analyzed. In the transgenic lines of $A$. thaliana, Gbwril resumed the seed weight, seed area, and surface morphology to the wild type. Gbwril transformation rescued the wrinkled phenotype of wri1-3 mutants by resuming the expression of fatty acid biosynthesis genes biotin carboxyl carrier protein isoform 2 (bccp2) and keto-ACP synthase 1 (kas 1 ). Moreover, the seedling development of transgenic lines on non-sucrose medium demonstrated that the Gbwril was able to regulate the supply of sucrose for normal seedling establishment. Our results showed that the transformation of Gbwril in A. thaliana wri1-3 mutant was able to complement wri1-3 impaired phenotype. Thus, Gbwril is involved in cotton fiber development and fatty acid biosynthesis in seeds. (C) 2021 Friends Science Publishers
\end{abstract}

Keywords: Gossypium barbadense; Transcription factor; Ectopic expression; Fatty acid biosynthesis

\section{Introduction}

Oil seed crops play important role in agricultural economy next to cereals. Cotton being the most important oil seed crop is considered as valuable resource for bioenergy ( $\mathrm{Li}$ et al. 2016). Identification and manipulation of the genetic factors and molecular networks controlling the fatty acid synthesis may result in significant increase in oil yield (LiBeisson et al. 2013). The biosynthesis of fatty acids is a multistep process and not only involves series of genes but also highly related to other physiological processes like glycolysis, oxidative phosphorylation and protein synthesis (Liu et al. 2018). Thus, manipulation of single gene is not considered as the feasible choice. However, transcription factors involved in regulatory functions associated with lipid metabolism might be the significant tool to address the bottleneck of increasing the seed oil content (Wang et al. 2007; Zhang et al. 2016).
WRINKLED1 is a plant specific, ethylene-responsive transcription factor belonging to APETALA2 family containing two AP2 DNA-binding domains. The widely studied WRINKLED1 from Arabidopsis thaliana (ATWRI1; At3g54320) binds with the promoters of genes involved in glycolysis and fatty acid biosynthesis (Marchive et al. 2014; Liu et al. 2018). Two null alleles of wril i.e., wril-3 (N585693) and wril-4 (N508559) were found in SALK collection (Alonso et al. 2003) which displayed TDNA insertions in the $5^{\text {th }}$ and $6^{\text {th }}$ introns of wril respectively. Transcriptome profile comparison showed the reduced expression of the genes involved in fatty acid and glycolytic enzymes in the wril-1 mutant seeds compared to wild type (Liu et al. 2018).

Seeds of loss-of-function mutant Atwril display 80\% reduction in triacyl glyceride (TAG) accumulation and increase in the sucrose level as compared to wild type, indicating that Atwril is a regulator for allocating carbon

To cite this paper: Imran M, S Bano, A Manzoor, M Azeem, T Rehman, S Yousaf, M But, T Hussain, U Qaisar (2021). Ectopic expression of fiber related Gbwril complements seed phenotype in Arabidopsis thaliana. Intl J Agric Biol 25:123-130 
towards fatty acid or sucrose biosynthesis pathway. The splicing mutant showed a compromised glycolysis due to which the developing seeds were unable to convert sucrose into TAGs (Wu et al. 2014). Due to decreased fatty acid content these mutants show impaired seedling germination (Ma et al. 2015).

WRI1 affects seed oil production in plants of Zea mays (Pouvreau et al. 2011), Brassica napus (Liu et al. 2010; Wu et al. 2014) Brachypodium distachyon (Yang et al. 2015), Brassica juncea (Bhattacharya et al. 2016) and Lepidium campestre (Ivarson et al. 2017). Zang et al. (2018) have conducted genome wide identification of wrinkled1 genes from G. hirsutum, G. barbadense, G. arboreum and $G$. raimondii species and have confirmed that the ectopic expression of wrila gene from $G$. hirsutum can rescue the seed phenotype in wril-7 mutant of A. thaliana. G. hirsutum was transformed to produce Ghwril over expressing lines and showed enhanced lipid content in seeds (Liu et al. 2018).

Some studies have shown that WRINKLEDl gene from cotton is involved in cotton fiber development (Qu et al. 2012; Qaisar et al. 2017). The silencing of wril in $G$. hirsutum enhanced fiber length and reduced oil content of seed, suggesting its positive correlation with fatty acid biosynthesis and negative with fiber elongation $(\mathrm{Qu}$ et al. 2012). All of the above studies were carried out with only G. hirsutum wril genes. However, the transcriptional profile of wril in elite fiber producing cotton ( $G$. barbadense) and G. hirsutum exhibited marked differences (Qaisar et al. 2017). The expression of wril was significantly higher in $G$. barbadense as compared to G. hirsutum and G. arboreum (Qaisar et al. 2017) indicating a positive correlation with fiber length. To date no direct information is available on involvement of any wril of $G$. barbadense in fatty acid biosynthesis. Thus, a study was designed to investigate the possible role of GbWRI1 in seed development and biosynthesis of fatty acids in model plant $A$. thaliana. We transformed Gbwril in loss of function mutant SALK_085693 of A. thaliana and performed morphological and molecular studies. As the transcription factor overexpression may result in some unwanted pleiotropic effects on certain morphological attributes in addition to expected increase of seed oil content, so its expression in model plant A. thaliana should be investigated. Therefore, the aim of the study was to clone the Gbwril transcription factor gene from $G$. barbadense and to raise transgenic lines overexpressing this gene. The expected results might lead to the potential modulation of $G b W R I 1$ over expression directly in cotton in order to get crop with better fiber quality and increased oil contents.

\section{Materials and Methods}

\section{Plant materials and growth conditions}

The experiment was conducted in research area of School of Biological Sciences, Punjab University, Lahore.
Arabidopsis thaliana wild type (Col-0) and wri1-3 mutant (SALK_085693) seeds were obtained from Arabidopsis Biological Resource Center, Ohio State University, OH, U.S.A. Seeds were surface sterilized with nascent $\mathrm{Cl}_{2}$ produced as a result of reaction between commercial bleach and concentrated $\mathrm{HCl}$ and grown on half-strength $\mathrm{MS}$ medium (Sigma, St. Louis, Missouri, United States) containing $2 \%$ sucrose and $0.7 \%$ agar. Plates were incubated in darkness for 2 days at $4^{\circ} \mathrm{C}$ and were placed in a plant growth chamber at $17-20^{\circ} \mathrm{C}$ in a light/dark period of $16 / 8 \mathrm{~h}$. After 10 days, the seedlings were transferred to soil in the growth chamber under the same conditions. Plants were watered regularly until flowers transformed to siliques. The whole plants were carefully bagged, dried and subjected to seed collection. The collected seeds were dried for further couple of days at $37^{\circ} \mathrm{C}$.

\section{Sequence retrieval, multiple sequence alignment and structure analysis}

Nucleotide sequence of probe (GhiAffx.60562.1.S1_at) on Affymetrix cotton genome array (Thermofisher Scientific) was retrieved from www.affymetrix.com as this probe showed involvement in fiber development (Qaisar et al. 2017). Full length sequence was retrieved by blasting the sequence of fiber related probe against Gossypium barbadense genome at cottonGen database (Yu et al. 2014). This sequence was named G. barbadense wril (Gbwril) due to homology with wrinkledl transcription factor. Full length Gbwril was isolated from G. barbadense var. Bar $14 / 5$ cDNA as described (Qaisar et al. 2017) using genespecific primers (Table 1) and was sequenced. Atwril sequence was retrieved from The Arabidopsis Information Resource (TAIR). Homologues of Gbwril in tetraploid cotton $G$. barbadense were identified through blastn using Gbwril as query against the G. barbadense HAU v2.0 CDS $(109,778)$ genome sequence database (Yu et al. 2014). The online translate tool (www.expasy.org) was used to predict the aminoacid sequences of WRI1 homologues. ClustalX version 2.0 was used to perform multiple sequence alignments of all identified wril homologues. Gene structures of all the wril homologues in G. barbadense were generated using the Gene Structure Display Server. Homology model building of GbWRI1 proteins was done by uploading the amino acid sequence on SWISS-MODEL for structure prediction and homology building. 3-D structure of target protein was done based on sequence alignment between the target protein and the template structure.

\section{Construct preparation and transformation in A. thaliana}

RNA was extracted from $G$. barbadense fibers at 17 days post anthesis (DPA) as previously described (Qaisar et al. 2017). The cDNA was synthesized and amplified through polymerase chain reaction (PCR), 
cloned into pTG19-T PCR cloning vector (Vivantis, Selangor Darul Ehsan, Malaysia) and confirmed by sequencing. Primers used for Gbwril amplification were SF and WRIFCR1 (Table 1). After introducing the adaptors for recognition sites of restriction enzymes NcoI at 5' and PmlI at 3' termini, Gbwril was cloned into pCambia 1301 plant binary vector between CAMV $35 \mathrm{~S}$ promoter and NOS terminator. The recombinant plasmid (pSAM2) was electroporated into Agrobacterium tumefaciens strain LBA4404 and then transformed into A. thaliana by the floral dip method (Zhang et al. 2006). Transgenic plants were selected by growing them on MS medium containing hygromycin.

\section{Molecular confirmation of transgenic lines}

For the confirmation of integration of Gbwril in transgenic lines, genomic DNA was extracted from A. thaliana leaf tissues of ten days old transformed lines and untransformed control plants using GeneAll ${ }^{\circledR}$ Exgene $^{\mathrm{TM}}$ plant SV mini kit (Lisbon, Portugal) according to manufacturer's instructions. PCR was performed using sequence specific primers for Gbwril SF and wriR520 (Table 1). PCR product was visualized using agarose gel electrophoresis.

\section{Quantitative real-time PCR (qRT-PCR) analysis}

Shoots of $A$. thaliana transformed plants were harvested at silique formation stage, ground in liquid nitrogen and total RNA was extracted using RNeasy Plant Mini kit (Qiagen, Hilden, Germany) according to manufacturer's protocol. Genomic DNA contamination was removed using DNase I (Qiagen, Hilden, Germany). First-strand cDNA synthesis was performed using Reverse Transcriptase (Promega, Madison, Wisconsin, United States)). After optimization of gene specific primers (Table 1), relative expression of wrinkled1, biotin carboxyl carrier protein isoform 2 (bccp2) and keto-ACP synthase 1 (Kas 1$)$ was measured in transformants, wild type and wri1-3 mutant using real time RT-PCR as previously described (Qaisar et al. 2013, 2017). At least three biological replicates and three technical replicates for each genotype were used. A. thaliana actin gene (Table 1) served as endogenous control in these studies.

\section{Determination of seed weight, size and scanning electron microscopy}

Weight of hundred seeds from wild type (Col-0), wri1-3 mutant (SALK_085693) and transgenic A. thaliana lines was measured using a microbalance. The size of the seeds was determined by measuring the length and width of the seeds under a Light microscope (Optika Vision Lite Ponteranica, Italy) and using the software, Image $\mathbf{J}$ (https://imagej.nih.gov/ij/). The samples were mounted on carbon conductive tape pasted on aluminum sample holder and loaded into Scanning electron microscope (JOEL JSM-
6480LV, Freising, Germany) at $20 \mathrm{kV}$ for surface/morphology analysis.

\section{Statistical analysis}

One-way ANOVA and Bonferroni's multiple comparison test were performed in GraphPad Prism 5 software to evaluate the statistical significance of differences observed between wild type, wril-3 mutants and transformed lines expressing Gbwril.

\section{Results}

Gene structure and protein analysis of Gbwril and its allelic isoforms in $G$. barbadense

G. barbadense is a tetraploid cotton (4X) harboring four alleles of wrinkled1. Alleles of Gbwril in G. barbadense (Gbar_D10G021070.1,

Gbar_A10G020800.1, Gbar_D13G000170.1 and Gbar_A13G000380.1) were identified using blastn at cottonGen against Gossypium barbadense (AD2) genome HAU_v2 (www.cottongen.org). Gbwril showed $99 \%$ amino acid sequence homology with Gbar_D10G021070.1. Gene models of Gbwril and its four alleles were used to analyze the resemblance and diversity of their exon and intron structures (Fig. 1).

Gbwril and its homologues harbored a 5'UTR, 3'UTR, six exons and five introns. Gbar_D10G021070.1 and Gbar_A10G020800.1 showed close homology with Gbwril while Gbar_D13G000170.1 and Gbar_A13G000380.1 were undersized at the C-terminal end of the gene. To investigate the amino acid sequence similarity and diversity among Atwril, Gbwril, and its homologues, multiple sequence alignment was performed. The comparison showed that Gbwril was 10 amino acids shorter than Atwril. While Gbar_D10G021070.1, Gbar_A10G020800.1, Gbar_D13G000170.1 and Gbar_A13G000380.1 were 2, 12, 58 and 68 amino acids shorter than Atwril, respectively. Interestingly, the alignment showed that the two APETALA2 DNA binding domains were highly conserved in all WRI1 proteins (Fig. 2).

Maximum protein and nucleotide sequence similarity was observed between Gbwril with Gbar_D10G021070.1 indicating that Gbwril is actually Gbar_D13G000170.1. Three dimensional structures based on homology modeling of the proteins of Atwril, Gbwril and its allelic forms were constructed (Fig. 3).

It was observed that DNA binding domains of Gbwril were identical to the $A$. thaliana wrinkled1 DNA binding domains in respect of their secondary structure. Both Gbwril and Atwril contain the same number of alpha helix and beta sheets. However, the helices had more turns and beta sheets were smaller in cotton isoforms in comparison with Atwril (Fig. 3). APETALA2 DNA binding domains which bind to the DNA through tryptophan and arginine residues in the beta sheets were conserved in beta sheets of both AtWRI1 and GbWRI1 domains. 
Table 1: Sequences and purpose of the primers used in this study

\begin{tabular}{|c|c|c|c|c|}
\hline Primer Pair & 5'-3' sequence & Product (bp) & Accession number & Objective \\
\hline $\mathrm{SF}$ & GATCAATCAGAGAAACCCAAGG & 1300 & Gbar_D10G021070 & wrinkledl full gene amplification \\
\hline WRIFCR1 & TTAAACAGAGTAGTTACAAGAAACC & & & \\
\hline SF & GATCAATCAGAGAAACCCAAGG & 351 & Gbar_D10G021070 & Gbwril confirmation in transgenic lines \\
\hline wriR520 & TGTCACTTTCTTCATTTCTTCСATCTCTTT & & & \\
\hline Arabi actin F & TGATTGGTATGGAGAATGCTGGTAT & 159 & AT2G37620 & Housekeeping gene for normalization of \\
\hline Arabi actin $\mathrm{R}$ & CAGTGATCTCTTTGCTCATTCTGTC & & & gene expression. \\
\hline $\operatorname{kas} 1 \mathrm{~F}$ & СТTCTCTGCCTTCAAACCCTGA & 204 & AT5G46290 & Expression analysis of kas 1 \\
\hline kaslR & TTCCGCAGACAAGAGAGAGAAC & & & \\
\hline bccp $2 \mathrm{~F}$ & GGTTTCGGATTTCTGCTTGTCG & 132 & AT5G15530 & Expression analysis of $b c c p 2$ \\
\hline bccp $2 \mathrm{R}$ & GACAGAGAGCCAGGATTGAGAG & & & \\
\hline
\end{tabular}

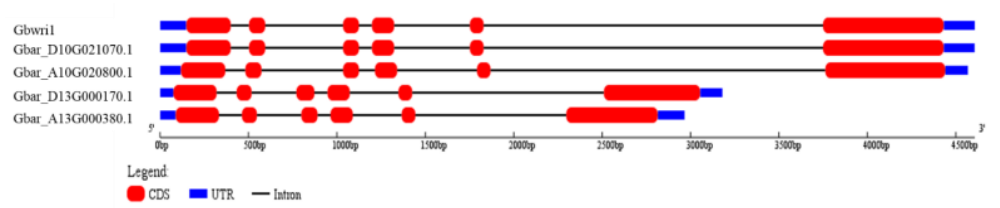

Fig. 1: Gene structures of Gbwril and its homologues in tetraploid cotton G. barbadense showing distribution of intronic and exonic regions

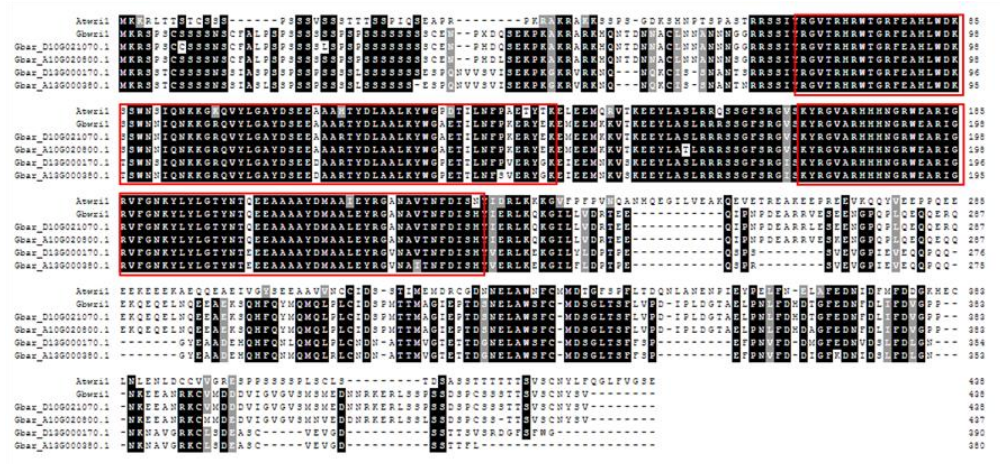

Fig. 2: Protein sequence alignment of Atwril, Gbwril and wril homologues in G. barbadense. Identical residues are shaded black, similar residues are shaded gray while AP2 domains are indicated by red boxes

\section{A. thaliana mutant and gene complementation}

Homology of Atwril and Gbwril in gene structure (Fig. 1 and 4), amino acid sequence (Fig. 2), 3D structure (Fig. 3) and DNA binding domains suggests functional homology between two homologues. Although Gbwril is involved in fiber development and Atwril plays an important role in seed filling and fatty acid biosynthesis. In order to test whether Gbwril can complement Atwril, a null allele of wril namely, wri1-3 (N585693) was identified in the SALK collection displaying T-DNA insertions in the $5^{\text {th }}$ intron of wril (Fig. 4).

We cloned Gbwril in pCambia1301 plant expression vector between cauliflower mosaic virus (CaMV) $35 \mathrm{~S}$ promoter and nopaline synthase (NOS) terminator (Fig. 4A) and transformed in wri1-3 mutant of $A$. thaliana using Agrobacterium mediated transformation.

\section{Integration and expression of Gbwril in A. thaliana}

To confirm the integration of Gbwril in transgenic lines of
A. thaliana, PCR was carried out with genomic DNA templates using gene-specific primers for Gbwril sequence. Agarose gel electrophoresis confirmed the presence of $351 \mathrm{bp}$ product in $\mathrm{A} 1, \mathrm{~A} 3$ and $\mathrm{A} 5$ transgenic lines of $A$. thaliana harboring Gbwril cDNA (Fig. 5A). G. barbadense cDNA was used as a positive control. To check the transcription of Gbwril in the transgenic lines, mRNA levels were checked in the transformed and untransformed lines of A. thaliana using real-time RT-PCR. We found that A1 and A3 showed significantly higher transcript levels (6and 9-fold respectively) of Gbwril as compared to untransformed mutant (Fig. 5B).

\section{Gbwril resumed the expression of fatty acid biosynthesis} genes and seed weight in wri1-3 mutant of $A$. thaliana

We checked the expression of bccp2 (AT5G15530) and kasl (AT5G46290) in the mutant and transgenic lines in order to see whether Gbwril has resumed the function of Atwril by enhancing the expression of these target genes. Transcript accumulation was dropped 15-fold in the mutant 

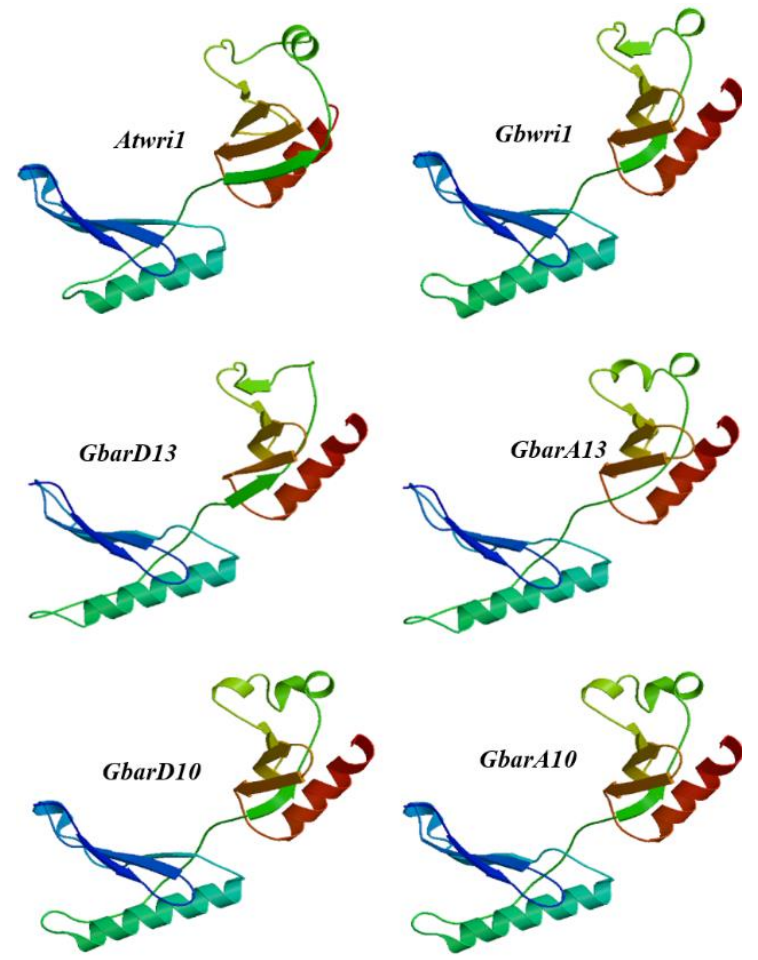

Fig. 3: Three-dimensional protein structures of Atwril, Gbwril and wril homologues in $G$. barbadense.

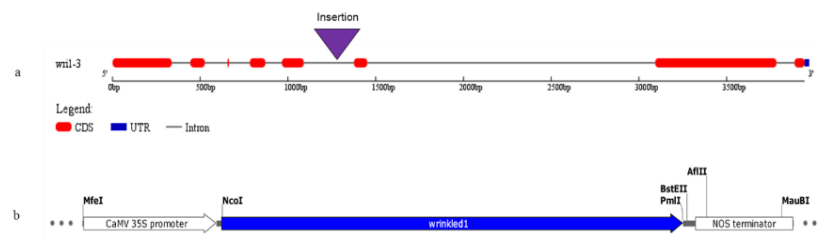

Fig. 4: Complementation of wri1-3 mutant of A. thaliana with Gbwril expression cassette. (A) Gene model of $A$. thaliana wri1-3 mutant showing the site of T-DNA insertion. (B) T-DNA element of pSAM2 plasmid harboring Gbwril coding region from $G$. barbadense

as compared to wild type while transformation with Gbwril in A3 line partially resumed the expression to a level half than the wild type (Fig. 6A). While in A1 line, the expression increased non-significantly as compared to wri13 mutant. Transposon insertion in wril-3 mutant reduced the expression of kasl gene 8.5 fold as compared to wild type. This reduction in expression was compensated by transformation of functional Gbwril, up to 3 fold in A1 and 4 fold in A3 line in comparison with mutant (Fig. 6B).

Ectopic expression of Gbwril rescued the seed morphology of wri1-3 mutant of $A$. thaliana

Seeds of T3 homozygous progeny of transgenic lines were investigated and compared with wild type and wri1-3 mutant. Microscopic observation of the mature seeds
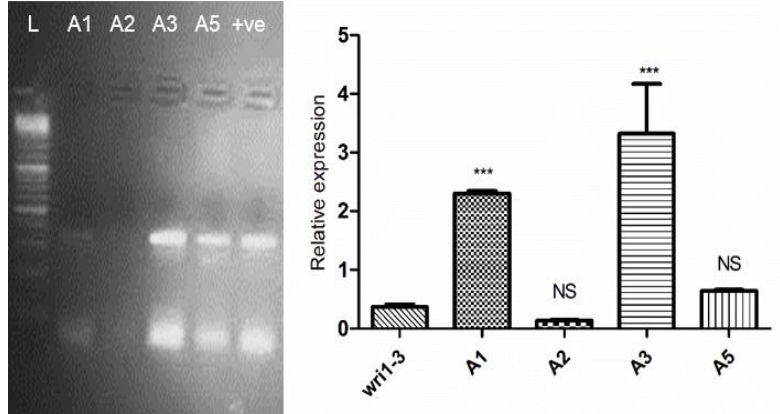

Fig. 5: Confirmation of transgene in transgenic lines of $A$. thaliana. (A) Amplification of Gbwril in DNA isolated from transgenic lines of A. thaliana. (B) Transcript levels of transgene in transformed lines. At least three biological replicates of each genotype were used. Error bars represent standard deviation, ns indicates $P$-value is greater than 0.05 . *** means $P$-value is less than 0.001
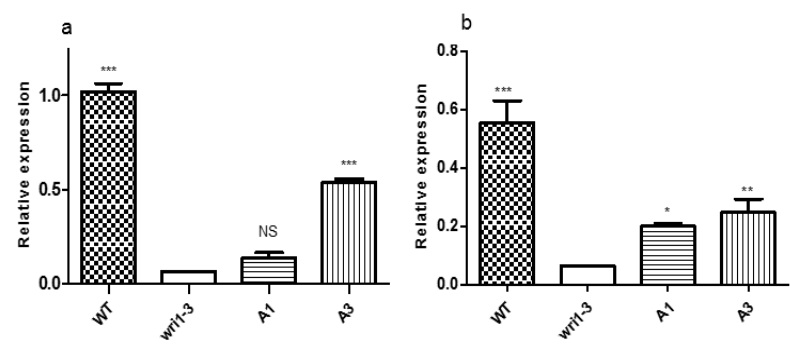

Fig. 6: Transcription of WRI1 target genes in transgenic and parent lines (A) bccp2 gene (B) Kasl gene. At least three biological replicates of each line were used for expression analysis. NS, $P>0.05$; ***, $P<0.001$; **, $P<0.01$; *,$P<0.05$

showed that wri1-3 mutant seeds were much smaller in size as compared to wild type while a reversion of wrinkled phenotype was observed in transgenic lines (Fig. 7A).

The seed surface analysis indicated that A3 line showed complete reversion while A1, A5 and A2 exhibited partial reversion (Fig. 7B). In addition to seed surface, further seed traits were observed in transgenic lines. Examination of seed weight (per 100 seeds) showed that ectopic expression of Gbwril significantly increased the seed weight in transgenic lines (Fig. 4B). We observed that the seed weight was increased by $30.9 \%, 24.2 \%, 19.3 \%$ and $19.3 \%$ in transgenic lines (A1, A2, A3 and A5) respectively as compared to mutant (Fig. 8A). In agreement with increased seed weight, seed area was also significantly increased as compared to wild type. The wri1-3 mutant showed a 26 percent reduction in seed area as compared to wild type seeds which was resumed in transgenic lines to the wild type (Fig. 8B).

Impaired germination and seed phenotype of wri1-3 mutant is rescued by Gbwril

During early plant growth, TAG reserves are broken down 


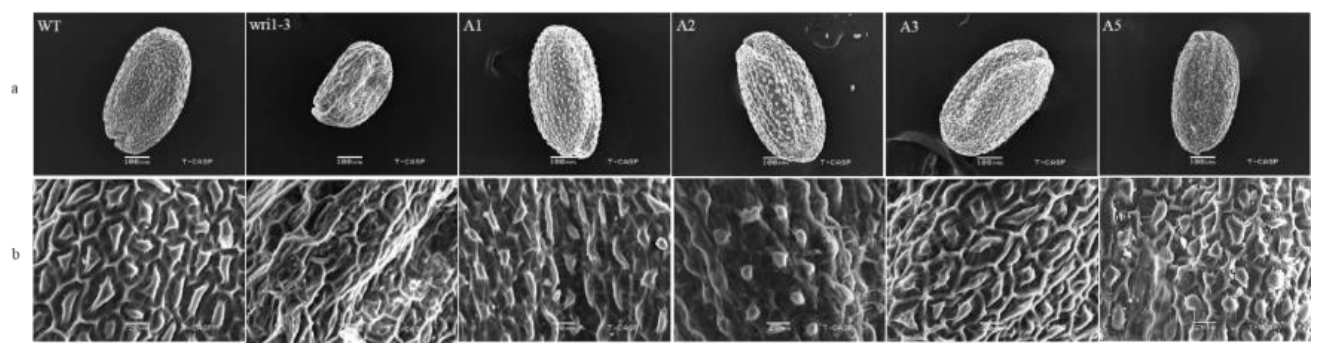

Fig. 7: Comparison of wildtype (WT), mutant (wri1-3) and transgenic lines (A1, A2, A3 and A5) of Arabidopsis thaliana. (A) Mature seeds. (B) Scanning electron microscopic images of seed surfaces
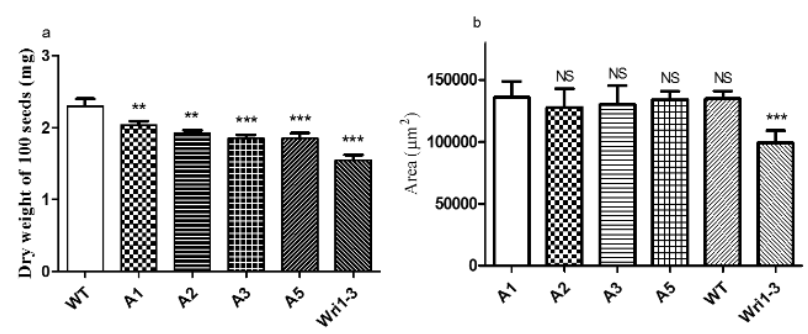

Fig. 8: Ectopic expression of Gbwril rescues seed of Arabidopsis thaliana. A.100 Seed dry weight and B, seed area. Length of the bar represents the mean value of triplicates. Error bars depict standard deviation. *** indicates $P$ value $<0.001$, ** $P$ value $<$ 0.01 and NS represents $P$ value $>0.05$.
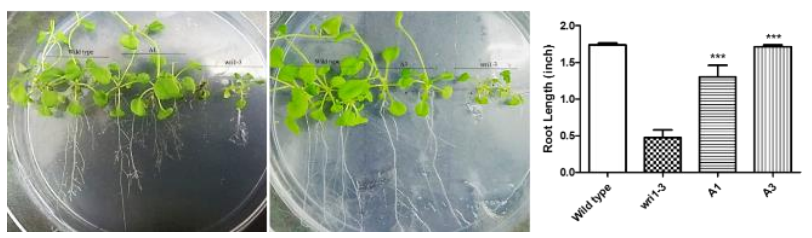

Fig. 9: Growth of wild type, mutant and transgenic lines (A1 and A3) of Arabidopsis thaliana on sucrose free medium. Error bars represent standard deviation

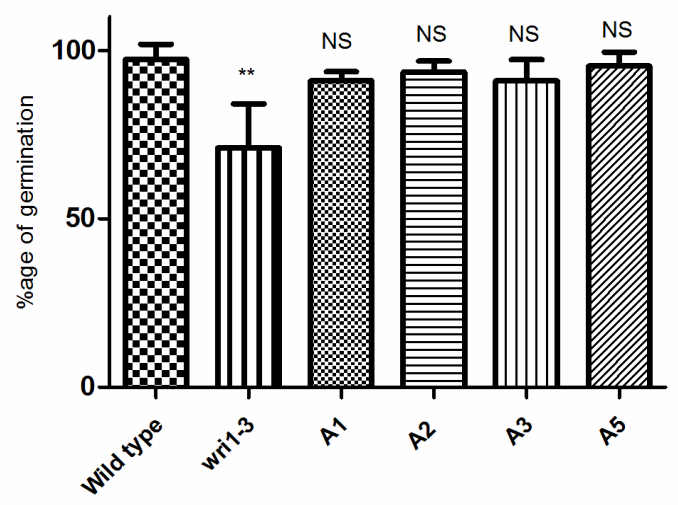

Fig. 10: Seed germination rate of wild type, wri1-3 mutant, and transgenic lines of Arabidopsis thaliana. NS represents $P>0.05$; ** $P<0.01$

into soluble sugar molecules which can be transported to the developing seedlings (Pritchard et al. 2002). The wrinkledl mutants of $A$. thaliana had reduced seed storage compounds required for normal seed development (Ma et al. 2013). Sucrose in the medium is required as building blocks and energy supply during wri1-3 seedling establishment. Due to decreased fatty acid content of wri1-3 mutants the seedling germination and establishment were impaired in the medium without sucrose. The wri1-3 mutant displays reduced seed germination and root development on sucrosefree medium (Fig. 9).

However, the transformation of Gbwril resumed seed germination and root growth to the wild type. Transgenic wri1-3 mutants expressing Gbwril developed normal seedlings on the medium lacking sucrose (Fig. 9). To further confirm the reduced seed germination in wri1-3 mutant, the plants were grown on MS agar medium. The wri1-3 mutants showed a $27 \%$ reduced seed germination compared to the wild type, while the germination of $\mathrm{A} 1$, A2, A3, and A5 transgenic lines was equivalent to the wild type (Fig. 10).

\section{Discussion}

WRINKLED1 protein is an APETAL2 like transcription factor that binds with the promoters of fatty acid biosynthesis and carbohydrate metabolism genes and is involved in regulation of oil accumulation during maturation of A. thaliana seeds (Junker et al. 2012). A WRI1-like gene from B. napus (BnWRII) showed $80 \%$ sequence identity with AtWRII and overexpression of which increased seed mass and oil content in transgenic $A$. thaliana (Liu et al. 2010; Kong et al. 2020). This loss of function mutant line produces wrinkled seeds and the transcript levels are severely reduced as compared to wild type (Junker et al. 2012). Like Atwril, the seed-expressed Ghwril orthologs have been observed regulating seed oil biosynthesis by not only complementing the wril-7 but also by increasing the seed oil content (Liu et al. 2018; Zang et al. 2019; Correaa et al. 2020). The involvement is further confirmed by the silencing of $G$. hirsutum wrinkledl gene which resulted in enhanced cotton fiber length and reduced fatty acid biosynthesis by partitioning the carbon flow $(\mathrm{Qu}$ et al. 2012). We have previously reported that $G$. barbadense, which possesses marked differences in cotton 
fiber morphology, revealed elevated expression of Gbwril transcription factor compared to G. hirsutum (Qaisar et al. 2017). Moreover, Gbwril expression in G. barbadense is positively correlated with fiber length in contrast with $G$. hirsutum WRINKLED1. This unusual behavior led us to further investigate the possible role of Gbwril seed development and biosynthesis of fatty acids in model plant A. thaliana.

In this study we inquired whether GbWRI1 which is more highly associated with higher fiber length might have evolved modified traits associated with this function. It was further investigated whether GbWRI1 could function in seed oil synthesis regulation or result in modified phenotypes regarding germination and seedling development. GbWRI1 can complement germination and seedling development phenotypes of wril in A. thaliana have not been studied previously. This is the first report highlighting the role of WRII allele of G. barbadense in A. thaliana.

WRI1 transcription factor induced fatty acid buildup leading to oil accumulation in seeds of $A$. thaliana and mutation of AtWRI1 failed to produce seed oil resulting in deformed seeds with wrinkled appearance, less surface area and dry weight (Junker et al. 2012; Wu et al. 2014). In wri13 loss of function mutant, expression of target genes of Atwril, i.e. bccp2 and kas1 is reduced leading to decreased fatty acid content, Where we report that ectopic expression of GbWRI1 in A. thaliana mutant (wri1-3) lead to overexpression of oil accumulating genes bccp-2 and kas-1 which effected seed weight, size, germination rate and seed morphology. The similar findings were observed in $G$. hirsutum where overexpression of Ghwril enhanced lipid content in seeds (Liu et al. 2018; Correaa et al. 2020). Like GhWRI1, GbWRI1 shows similarity in gene structure (Fig. 1 and 4), amino acid sequence (Fig. 2), 3D structure (Fig. 3) and DNA binding domains with AtWRI1. These results indicate that due to functional homology between two homologues, this GbWRI1 can rescue the wri1-3 mutant seed phenotype probably by regulating fatty acid biosynthesis.

Our study also revealed that transformation of GbWRI1 rescued the seed germination and root growth in wri1-3 mutants. During early plant growth, TAG reserves are broken down into soluble sugar molecules which can be transported to the developing seedlings (Pritchard et al. 2002). The wrinkledl mutants of $A$. thaliana had reduced seed storage compounds required for normal seed development (Ma et al. 2013). Sucrose in the medium is required as building blocks and energy supply during wri1-3 seedling establishment. Due to decreased fatty acid content of wri1-3 mutants the seedling germination and establishment were impaired in the medium without sucrose. GbWRI1 also regulates glucogenesis in the absence of sucrose, leading to seedling establishment (Fig. 9) which emphasizes on the role of WRII in sugar metabolisms. As cellulose constitutes $85-97 \%$ of dry weight of cotton fiber and sugar molecules serve as precursors for cellulose biosynthesis (Pattathil et al. 2015; Cosgrove 2018; Herger et al. 2019; Vaahtera et al. 2019; Rui and Dinneny 2020) so GbWRI1 regulates fiber development through regulating sugar metabolism. Moreover, GbWRI1 shows higher expression at later stages of fiber development (secondary cell wall biosynthesis) which predominantly are involved in cellulose deposition.

\section{Conclusion}

The novel Gbwril gene has been observed to be involved in the regulation of seed lipid content in $A$. thaliana. Overexpression of Gbwril in native cotton varieties can be studied with reference to cotton fiber improvement and enhancement of seed oil content. This gene may be the potential candidate to provide an opportunity of commercial use for breeding transgenic crops.

\section{Acknowledgements}

We acknowledge Higher Education Commission of Pakistan for providing funds for the research.

\section{Author Contributions}

MI performed experiments; SB performed experiments; AM data analysis; SY data analysis; TR bioinformatics; MB paper write-up; TH Scanning Electron Microscopy; Uzma Qaisar acquired funding, experiment design and performed manuscript writing

\section{References}

Alonso JM, AN Stepanova, TJ Leisse, CJ Kim, H Chen, P Shinn, DK Stevenson, J Zimmerman, P Barajas, R Cheuk, C Gadrinab, C Heller, A Jeske, E Koesema, C C Meyers, H Parker, L Prednis, Y Ansari, N Choy, H Deen, M Geralt, N Hazari, E Hom, M, Karnes, C Mulholland, R Ndubaku, I Schmidt, P Guzman, L Aguilar-Henonin, M Schmid, D Weigel, DE, Carter, T Marchand, E Risseeuw, D Brogden, A Zeko, WL Crosby, CC Berry, JR Ecker (2003). Genome-wide insertional mutagenesis of Arabidopsis thaliana. Science 301:653-657

Bhattacharya S, N Das, MK Maiti (2016). Cumulative effect of heterologous AtWRII gene expression and endogenous BjAGPase gene silencing increases seed lipid content in Indian mustard Brassica juncea. Plant Physiol Biochem 107:204-213

Correaa SM, AR Ferniede, Z Nikoloskie, Y Brotman (2020). Towards model-driven characterization and manipulation of plant lipid metabolism. Progr Lipid Res 80:1-4

Cosgrove DJ(2018). Diffuse growth of plantcell walls. Plant Physiol 176:16-27

Herger A, K Dünser, J Kleine-Vehn, C Ringli (2019) Leucine-rich repeat extension proteins and their role in cell wall sensing. Curr Biol 29:851-858

Ivarson E, N Leiva-Eriksson, A Ahlman, S Kanagarajan, L Bülow, LH Zhu (2017). Effects of overexpression of WRII and hemoglobin genes on the seed oil content of Lepidium campestre. Front Plant Sci 7 ; Article 2032

Junker A, G Mönke, T Rutten, J Keilwagen, M Seifert, TM N Thi, JP Renou, S Balzergue, P Viehöver, U Hähnel (2012). Elongation-related functions of LEAFY COTYLEDON1 during the development of Arabidopsis thaliana. Plant J 71:427-442 
Kong Q, Y Yang, PM Low, L Guo, L Yuan, W Ma (2020). The function of the WRI1-TCP4 regulatory module in lipid biosynthesis. Plant Signal Behav 2020; Article 1812878

Li-Beisson Y, B Shorrosh, F Beisson, MX Andersson, V Arondel, PD Bates, S Baud, D Bird, A DeBono, TP Durrett, RB Franke, IA Graham, K Katayama, AA Kelly, T Larson, JE Markham, M Miquel, I Molina, I Nishida, O Rowland, L Samuels, KM Schmid, H Wada, R Welti, C Xu, R Zallot, J Ohlrogge (2013). Acyl-lipid metabolism. Arabidopsis Book 8; Article e0161

Li C, Y Dong, T Zhao, L Li, E Yu, L Mei, MK Daud, Q He, J Chen, S Zhu (2016). Genome-wide SNP linkage mapping and QTL analysis for fiber quality and yield traits in the upland cotton recombinant inbred lines population. Front Plant Sci 7; Article 1356

Liu J, W Hua, G Zhan, F Wei, X Wang, G Liu, H Wang (2010). Increasing seed mass and oil content in transgenic Arabidopsis by the overexpression of wri1-like gene from Brassica napus. Plant Physiol Biochem 48:9-15

Liu Z, Y Zhao, W Liang, Y Cui, Y Wang, J Hua (2018). Over-expression of transcription factor GhWRII in upland cotton. Biol Plantarum 62:335-342

Ma W, Q Kong, M Grix, JJ Mantyla, Y Yang, C Benning, J B Ohlrogge (2015). Deletion of a C-terminal intrinsically disordered region of WRINKLED 1 affects its stability and enhances oil accumulation in Arabidopsis. Plant J 83:864-874

Ma W, Q Kong, V Arondel, A Kilaru, P Bates, A N Thrower, C Benning, BJ Ohlrogge (2013). WRINKLED1, A Ubiquitous regulator in oil accumulating tissues from Arabidopsis embryos to oil palm mesocarp. PLoS One 8; Article e68887

Marchive C, K Nikovics, A To, L Lepiniec, S Baud (2014). Transcriptional regulation of fatty acid production in higher plants: Molecular bases and biotechnological outcomes. Eur J Lip Sci Technol 116:1332-1343

Pattathil S, U Avci, T Zhang, CL Cardenas, MG Hahn (2015). Immunological approaches to biomass characterization and utilization. Front Bioeng Biotechnol 3; Article 173

Pouvreau B, S Baud, V Vernoud, V Morin, C Py, G Gendrot, JP Pichon, J Rouster, W Paul, PM Rogowsky (2011). Duplicate maize Wrinkled1 transcription factors activate target genes involved in seed oil biosynthesis. Plant Physiol 156:674-686

Pritchard SL, WL Charlton, A Baker, IA Graham (2002). Germination and storage reserve mobilization are regulated independently in Arabidopsis. Plant J 31:639-647
Qaisar U, F Akhtar, M Azeem, S Yousaf (2017). Studies on involvement of Wrinkled1 transcription factor in the development of extra-long staple in cotton. Ind J Genet Plant Breed 77:298-303

Qaisar U, L Luo, CL Haley, SF Brady, NL Carty, JA Colmer-Hamood, AN Hamood (2013). The pvc operon regulates the expression of the Pseudomonas aeruginosa fimbrial chaperone/usher pathway (cup) genes. PLoS One 8; Article e62735

Qu JY, YF Geng, YW Sun, SQ Gao, BP Zhang, W Chen, NH Chua (2012). Dissecting functions of KATANIN and WRINKLED1 in cotton fiber development by virus-induced gene silencing. Plant Physiol 160:738-748

Rui Y,JRDinneny(2020). A wall with integrity: Surveillance and maintenance of the plant cell wall under stress. New Phytol 225:1428-1439

Vaahtera L, J Schulz, T Hamann (2019). Cell wall integrity maintenance during plant development and interaction with the environment. Nat Plants 5:924-932

Wang HW, B Zhang, YJ Hao, J Huang, AG Tian, Y Liao, JS Zhang, SY Chen (2007). The soybean Dof-type transcription factor genes, GmDof4 and GmDof11, enhance lipid content in the seeds of transgenic Arabidopsis plants. Plant J 52:716-729

Wu XL, ZH Liu, ZH Hu, RZ Huang (2014). BnWRI1 coordinates fatty acid biosynthesis and photosynthesis pathways during oil accumulation in rapeseed. J Integr Plant Biol 56:582-593

Yang Y, J Munz, C Cass, A Zienkiewicz, Q Kong, W Ma, J Sanjaya, Sedbrook, C Benning (2015). Ectopic expression of WRINKLED1 affects fatty acid homeostasis in Brachypodium distachyon vegetative tissues. Plant Physiol 169:1836-1847

Yu J, S Jung, CH Cheng, SP Ficklin, T Lee, P Zheng, D Jones, RG Percy, D Main (2014). CottonGen: a genomics, genetics and breeding database for cotton research. Nucl Acids Res 42:1229-1236

Zang X, X Geng, L Ma, N Wang, W Pei, M Wu, J Zhang, J Yu (2019). A genome-wide analysis of the phospholipid: Diacylglycerol acyltransferase gene family in Gossypium. BMC Genomics 20; Article 402

Zang X, W Pei, M Wu, Y Geng, N Wang, G Liu, J Ma, D Li, Y Cui, X Li, L Zhang, J Yu (2018). Genome-Scale Analysis of the WRI-Like Family in Gossypium and Functional Characterization of GhWRIIa Controlling Triacylglycerol Content. Front Plant Sci 9; Article 1516

Zhang X, R Henriques, SS Lin, Qi W Niu, NH Chua (2006). Agrobacterium-mediated transformation of Arabidopsis thaliana using the floral dip method. Nat Protoc 1:641-646 